\title{
Soliton-Like Spherical Symmetric Solutions of the Nonlinear Spinor Field equations in General Relativity
}

\author{
Siaka Massou ${ }^{1}$, Alain Adomou ${ }^{1,2, *}$, Jonas Edou ${ }^{1}$ \\ ${ }^{1}$ Department of Theoretical Physics and Mathematics, University of Abomey-Calavi, Abomey-Calavi, Benin \\ ${ }^{2}$ National Higher Institute of Industrial Technology, University of Abomey, Abomey, Benin
}

\section{Email address:}

elietteadomou@gmail.com (A. Adomou)

\section{To cite this article:}

Siaka Massou, Alain Adomou, Jonas Edou. Soliton-Like Spherical Symmetric Solutions of the Nonlinear Spinor Field equations in General Relativity. International Journal of Applied Mathematics and Theoretical Physics. Vol. 5, No. 4, 2019, pp. 118-128. doi: 10.11648/j.ijamtp.20190504.14

Received: November 5, 2019; Accepted: November 28, 2019; Published:Published:December 24, 2019

\begin{abstract}
In this research work, we opt for the static spherical symmetric metric. Thus, taking into account the own gravitational field of elementary particles, we have obtained exact static spheric symmetric solutions of the nonlinear spinor and gravitational fields equations. The nonlinear terms in the spinor lagrangian density characterize the self-interaction of a spinor field. We have investigated in detail equations with power and polynomial nonlinearities. In this case, we have obtained exact regular solutions which have a localized energy density and limited total energy (soliton-like solutions) only if the mass parameter in the spinor field equations is equal to zero. In additional to this, the total charge and the total spin are bounded. We have also shown that in the linear case, soliton-like solutions are absent. But in the flat space-time, the obtained solutions are soliton-like configurations. Therefore, the proper gravitational field of elementary particles, the geometrical properties of the metric and the nonlinear terms in the lagrangian density play a crucial role in the purpose to get the regular solutions with localized energy density and limited total energy.
\end{abstract}

Keywords: Lagrangian, Metric, Invariant, At Space-time

\section{Introduction}

In many models elaborated in the pure science in order to describe the configuration of elementary particles, the gravitational theory is absent. We can cite for example super symmetry super strings (SUSY) model, the standar model (SM) and the theories of great unification (GUT). These models of elementary particles adequately describe obervations. However, these models postulate the value of particle masses and do not use the concept explaining relationship among these masses. The elementary particles have been extensively examined at the level of special relativity, firstly, in the context of Abelian theories and later in non-Abelian theories. In this case, the influence of gravitational theory is not taken into account. For more details, please, refer to [1]. In the research work entitled "Geometrization of electromagnetism in tetrad-spinconnection gravity", carried out by Nicodem J. Poplawski [2], it proved that the metric-affine lagrangian of Ponomarev and Obukhov for the unified gravitational and electromagnetic field is linear in the Ricci scalar and quadratic in the tensor of homothetic curvature. Applying the variational principle to the lagrangian with the tetrad and spin connection as dynamical variables, it is shown that the field equations are the Einstein-Maxwell equations if the electromagnetic potentiel is related to the trace of spin connection. It also proved that as in the Ponomarev-Obukhov [3] formulation, the generally covariant Dirac lagrangian gives rise to the standard spinor source for the Einstein-Maxwell equations, while the spinor field obeys the nonlinear Heisenberg-Ivanenko equation with 
the electromagnetic coupling. This author generalizes that formulation to spinors with arbitrary electric charges. The exact self-consitent particle-like solutions to the equations governing the interacting system of nonlinear scalar and electrodynamics fields in General relativity are obtained and investigated by the reseach group composed of Yu.P.Rybakov, G.N.Shikin and B.Saha [4]. Their research highlights that the equations of motion admit a special kind of solutions with sharp boundary known as droplet. For these solutions, the physical fields vanish and the space-time is flat outside of the critical sphere or cylinder. Note that the mass and the electric charge of these configurations are zero. In a remarkable research work on soliton, A. Adomou and G.N.Shikin [5] have obtained exact plane-symmetric solutions to the spinor field equations with nonlinear terms which are arbitrary functions of the invariant $S=\bar{\psi} \psi$. Taking into account the proper gravitational field of elementary particles, they have studied in detail equations with power and polynomial nonlinearities. Later, the research group composed of V.Adanhoumè, A.Adomou, F.P.Codo and M.N.Hounkonnou [6] have done an extension of the previous work. In their research work entitled" Nonlinear Spinor Field Equations in Gravitaional Theory: Spherical Symmetric Soliton-Like Solutions", they have obtained the spherical soliton-like solutions. They have also studied the regularity properties as well as the asymptotic behavior of the energy and charge densities. The plane-symmetric solitons of spinor and scalar fields are studied by B.Saha and G.N.Shikin [7]. They have considered a system of nonlinear spinor and scalar fields with minimal coupling in general relativity. The nonlinear term in the spinor field is given by an arbitrary function depending on the bilinear spinor forms $S=\bar{\psi} \psi$ and $P=\left(i \bar{\psi} \gamma^{5} \psi\right)$. As for the scalar lagrangian, it is chosen as an arbitrary function of the scalar invariant $\Omega=\phi_{, \alpha} \phi^{, \alpha}$ that becomes linear when $\Omega \longrightarrow 0$. The spinor and scalar fields in question interact with each other by means of a gravitational field. The gravitational field is given by a plane-symmetric metric. They have obtained exact plane-symmetric solutions to the gravitational, spinor and scalar field equations. They have also investigated the role of gravitational field in the formation of the configurations with limited total energy, spin and charge. In general, they have proved that the choice of spinor field nonlinearity can lead to the elimination of scalar field contribution to the metric functions, but its contribution to the total energy unaltered. A.Adomou, Jonas Edou and Siaka Massou [8] have obtained exact static plane-symmetric solutions to the spinor field equations with nonlinear terms which are arbitrary functions of invariant $I_{p}=P^{2}=\left(i \bar{\psi} \gamma^{5} \psi\right)^{2}$, taking into account their own gravitational field. They have shown that the initial set of the Einstein and spinor field equations with a power-law nonlinearity have regular solutions with a localized energy density of the spinor field only if $m=0$ ( $\mathrm{m}$ is the mass parameter in the spinor field equations). Equations with power and polynomial nonlinearities are studied in detail. In this case a soliton-like configuration has negative energy. They also obtained exact static plane-symmetric solutions to the above spinor field equations in flat space-time. It is proved that in this case soliton-like solutions are absent. The same authors [9] pursue the precedent work in a remarkable paper appeared in November 20th 2019. They have worked on the static spherical symmetric metric. In this metric, they have obtained spherical symmetric soliton-like solutions to the spinor field equations with nonlinear terms which are arbitrary functions of $I_{p}=P^{2}=\left(i \bar{\psi} \gamma^{5} \psi\right)^{2}$, taking into account their proper gravitational field. Equations with power and polynomial nonlinearities are investigated in detail. It is shown that the initial set of the Einstein and spinor field equations with a power-law nonlinearity have regular solutions with a localized energy density of the spinor field only if $m=0$ ( $\mathrm{m}$ is the mass parameter in the spinor field equations). In this case a soliton-like configuration has negative energy. They have also obtained exact static symmetric solutions to the above spinor field equations in flat space-time. It is proved that soliton-like solutions exist in this case. They have examined the role of gravitational field in the formation of the field configurations with limited total energy, spin and charge.

The rest of the paper is organized as follows. Section 2 deals with basic equations and their general solutions which represents the methods. We get exact spherical-symmetric solutions of Einstein and spinor field equations in general relativity theory, considering the proper gravitational field of elementary particles in the section 3 . Section 4 addresses the analysis of the results by choosing a concrete form of nonlinear terms in the spinor lagrangian density. Concluding remarks are outlined in section 5 .

\section{Methods: Einstein and Spinor Field Equations}

We start this section with the general form of the lagrangian density for the self-consistent system of spinor and gravitational fields:

$$
\begin{aligned}
L & =\frac{R}{2 \chi}+L_{S p} \\
& =\frac{R}{2 \chi}+\frac{i}{2}\left(\bar{\psi} \gamma^{\mu} \nabla_{\mu} \psi-\nabla_{\mu} \bar{\psi} \gamma^{\mu} \psi\right)-m \bar{\psi} \psi+L_{N}(1)
\end{aligned}
$$

where $L_{N}$ is the nonlinear part of the spinor field lagrangian; $\mathrm{R}$ is the scalar curvature, $\chi=\frac{8 \Pi G}{C^{4}}$ is Einstein's gravitational constant and G is Newton's gravitational constant. $L_{N}=$ $G\left(I_{v}\right)$ is an arbitrary function depending on the invariant function $I_{v}=S^{2}+P^{2}=\left(\bar{\psi} \gamma^{\mu} \psi\right) g_{\mu \nu}\left(\bar{\psi} \gamma^{\nu} \psi\right)$.

In the present analysis, the gravitational field is defined by the static spherical symmetric metric under the form:

$$
d s^{2}=e^{2 \gamma} d t^{2}-e^{2 \alpha} d \xi^{2}-e^{2 \beta}\left[d \theta^{2}+\sin ^{2} \theta d \varphi^{2}\right] .
$$

The signature of the metric (2) is considered as $(+1,-1,-1$, $-1)$ and $c=1$ as the unit of the speed of light. The metric 
functions $\alpha, \beta$ and $\gamma$ are some functions depending only on $\xi=\frac{1}{r}$ where $\mathrm{r}$ stands for the radial component of the spherical symmetric metric. They obey the coordinate condition given

by the following expression

$$
\alpha=2 \beta+\gamma .
$$

The matricial form of the metric tensor $g_{\mu \nu}$ associated to the metric (2) is

$$
\left[g_{\mu \nu}\right]=\left(\begin{array}{cccc}
e^{2 \gamma} & 0 & 0 & 0 \\
0 & -e^{2 \alpha} & 0 & 0 \\
0 & 0 & -e^{2 \beta} & 0 \\
0 & 0 & 0 & -e^{2 \beta} \sin ^{2} \theta
\end{array}\right)
$$

Varying (1) with respect to the metric tensor $g_{\mu \nu}$, we obtain the Einstein's field equations in the metric (2) under the condition (3) having the forms [14]

$$
\begin{gathered}
G_{0}^{0}=e^{-2 \alpha}\left(2 \beta^{\prime \prime}-2 \gamma^{\prime} \beta^{\prime}-\beta^{2}\right)-e^{-2 \beta}=-\chi T_{0}^{0} \\
G_{1}^{1}=e^{-2 \alpha}\left(2 \beta^{\prime} \gamma^{\prime}+\beta^{2}\right)-e^{-2 \beta}=-\chi T_{1}^{1} \\
G_{2}^{2}=e^{-2 \alpha}\left(\beta^{\prime \prime}+\gamma^{\prime \prime}-2 \beta^{\prime} \gamma^{\prime}-\beta^{\prime 2}\right)=-\chi T_{2}^{2} \\
G_{2}^{2}=G_{3}^{3}, \quad T_{2}^{2}=T_{3}^{3}
\end{gathered}
$$

where prime denotes differentiation with respect to the spatial variable $\xi$ and $T_{\nu}^{\mu}$ is the energy-momentum tensor of the spinor field.

From the lagrangian (1), applying the variational principle, we obtain the spinor field equations for the functions $\psi$ and $\bar{\psi}$ as follows

$$
\begin{aligned}
& i \gamma^{\mu} \nabla_{\mu} \psi-m \psi+2 S \frac{\partial G}{\partial I} \psi+2 i P \frac{\partial G}{\partial J} \gamma^{5} \psi=0 \\
& i \nabla_{\mu} \bar{\psi} \gamma^{\mu}+m \bar{\psi}-2 S \frac{\partial G}{\partial I} \bar{\psi}-2 i P \frac{\partial G}{\partial J} \gamma^{5} \bar{\psi}=0
\end{aligned}
$$

with $I=S^{2}$ and $J=P^{2}$.

The general form of the metric energy-momentum tensor of the spinor field is

$$
T_{\mu}^{\nu}=\frac{i}{4} g^{\nu \rho}\left(\bar{\psi} \gamma_{\mu} \nabla_{\nu} \psi+\bar{\psi} \gamma_{\nu} \nabla_{\mu} \psi-\nabla_{\mu} \bar{\psi} \gamma_{\nu} \psi-\nabla_{\nu} \bar{\psi} \gamma_{\mu} \psi\right)-\delta_{\mu}^{\nu} L_{S} p
$$

Using the spinor field equations for the functions $\psi$ and $\bar{\psi}, L_{S p}$ becomes

$$
L_{S_{P}}=\frac{1}{2} \bar{\psi}\left(i \gamma^{\mu} \nabla_{\mu} \psi-m \psi\right)-\frac{1}{2}\left(i \nabla_{\mu} \bar{\psi} \gamma^{\mu}+m \bar{\psi}\right) \psi+G(S, P)=-2 I \frac{\partial G}{\partial I}-2 J \frac{\partial G}{\partial J}+G(S, P)=-2 I_{v} \frac{\partial G}{\partial I_{v}}+G(S, P)
$$

Taking into account (14), the nonzero components of the tensor $T_{\nu}^{\mu}$ are:

$$
\begin{gathered}
T_{0}^{0}=T_{2}^{2}=T_{3}^{3}=-L_{S p}=2 I_{v} \frac{\partial G(S, P)}{\partial I_{v}}-G(S, P) . \\
T_{1}^{1}=\frac{i}{2}\left(\bar{\psi} \gamma^{1} \nabla_{1} \psi-\nabla_{1} \bar{\psi} \gamma^{1} \psi\right)+2 I_{v} \frac{\partial G(S, P)}{\partial I_{v}}-G(S, P) .
\end{gathered}
$$

In flat space-time, the Dirac's matrices $\bar{\gamma}^{a}$ are determined by the following expressions 


$$
\bar{\gamma}^{0}=\left(\begin{array}{cc}
I & 0 \\
0 & -I
\end{array}\right), \quad \bar{\gamma}^{i}=\left(\begin{array}{cc}
0 & \sigma^{i} \\
-\sigma^{i} & 0
\end{array}\right), \quad \bar{\gamma}^{5}=\gamma^{5}=\left(\begin{array}{cc}
0 & -I \\
-I & 0
\end{array}\right)
$$

where I is the two order unity matrice and $\sigma^{i}$ are Pauli's matrices defined as follows

$$
\sigma^{1}=\left(\begin{array}{cc}
0 & 1 \\
1 & 0
\end{array}\right), \quad \sigma^{2}=\left(\begin{array}{cc}
0 & -i \\
i & 0
\end{array}\right), \quad \sigma^{3}=\left(\begin{array}{cc}
1 & 0 \\
0 & -1
\end{array}\right)
$$

In curved space-time, the Dirac's matrices $\gamma^{\mu}$ are defined in the following way.

Using the equalities

$$
\begin{aligned}
\gamma_{\mu}(\xi) & =e_{\mu}^{a}(\xi) \overline{\gamma_{a}} \\
g_{\mu \nu}(\xi) & =e_{\mu}^{a}(\xi) e_{\nu}^{b}(\xi) \eta_{a b}
\end{aligned}
$$

where $\eta_{a b}=\operatorname{diag}(1,-1,-1,-1)$ is Minkowski's metric [15, 16, 17], $e_{\mu}^{a}(\xi)$ are tetradic 4-vectors, we obtain

$$
\gamma^{0}=e^{-\gamma} \bar{\gamma}^{0}, \quad \gamma^{1}(x)=e^{-\alpha} \bar{\gamma}^{1}, \quad \gamma^{2}=e^{-\beta} \bar{\gamma}^{2}, \quad \gamma^{3}=\frac{e^{-\beta} \bar{\gamma}^{3}}{\sin \theta}
$$

The general form of the spinor affine connection matrices is

$$
\Gamma_{\mu}(\xi)=\frac{1}{4} g_{\rho \mu}\left(\partial_{\mu} e_{\sigma}^{b} e_{a}^{\rho}-\Gamma_{\mu \sigma}^{\rho}\right) \gamma^{\delta} \gamma^{\sigma}
$$

where $\Gamma_{\mu \sigma}^{\rho}$ are Christofell symbols.

The expression (17) leads to

$$
\Gamma_{0}=-\frac{1}{2} e^{-2 \beta} \bar{\gamma}^{0} \bar{\gamma}^{1} \gamma^{\prime}, \quad \Gamma_{1}=0, \quad \Gamma_{2}=\frac{1}{2} e^{-\beta-\gamma} \bar{\gamma}^{2} \bar{\gamma}^{1} \beta^{\prime}, \quad \Gamma_{3}=\frac{1}{2}\left(e^{-\beta-\gamma} \bar{\gamma}^{3} \bar{\gamma}^{1} \beta^{\prime} \sin \theta+\bar{\gamma}^{3} \bar{\gamma}^{2} \cos \theta\right)
$$

In (9)-(11), $\nabla_{\mu}$ is the covariant derivative of spinor $[13,14] . \nabla_{\mu}$ is linked to the spinor affine connection matrices $\Gamma_{\mu}(\xi)$ by

$$
\nabla_{\mu} \psi=\frac{\partial \psi}{\partial \xi^{\mu}}-\Gamma_{\mu} \psi \quad \text { or } \quad \nabla_{\mu} \bar{\psi}=\frac{\partial \bar{\psi}}{\partial \xi^{\mu}}+\Gamma_{\mu} \bar{\psi}
$$

From (16) and (18), the Einstein's sommation gives

$$
\gamma^{\mu} \Gamma_{\mu}=-\frac{1}{2}\left(e^{-\alpha} \alpha^{\prime} \bar{\gamma}^{1}+\bar{\gamma}^{2} e^{-\beta} \cot \theta\right)
$$

Taking into account (19) and (20), the spinor field equations (9) and (10) lead to the following expressions

$$
\begin{aligned}
& i e^{-\alpha} \bar{\gamma}^{1}\left(\partial_{\xi}+\frac{1}{2} \alpha^{\prime}\right) \psi+\frac{i}{2} \bar{\gamma}^{2} e^{-\beta} \psi \cot \theta-(m-D) \psi+i E(S, P) \gamma^{5} \psi=0, \\
& i e^{-\alpha} \bar{\gamma}^{1}\left(\partial_{\xi}+\frac{1}{2} \alpha^{\prime}\right) \bar{\psi}+\frac{i}{2} \bar{\gamma}^{2} e^{-\beta} \bar{\psi} \cot \theta+(m-D) \bar{\psi}-i E(S, P) \gamma^{5} \bar{\psi}=0,
\end{aligned}
$$

where

$$
D(S, P)=2 S \frac{d G}{d I_{v}}, \quad E(S, P)=2 P \frac{d G}{d I_{v}}
$$

From (21), when $\psi(\xi)=V_{\delta}(\xi)$ with $\delta=1,2,3$, 4., we obtain the following system of equations

$$
V_{4}^{\prime}+\frac{1}{2} \alpha^{\prime} V_{4}-\frac{i}{2} e^{\alpha-\beta} V_{4} \cot \theta+i e^{\alpha}(m-D) V_{1}-E e^{\alpha} V_{3}=0,
$$




$$
\begin{aligned}
& V_{3}^{\prime}+\frac{1}{2} \alpha^{\prime} V_{3}+\frac{i}{2} e^{\alpha-\beta} V_{3} \cot \theta+i e^{\alpha}(m-D) V_{2}-E e^{\alpha} V_{4}=0, \\
& V_{2}^{\prime}+\frac{1}{2} \alpha^{\prime} V_{2}-\frac{i}{2} e^{\alpha-\beta} V_{2} \cot \theta-i e^{\alpha}(m-D) V_{3}+E e^{\alpha} V_{1}=0, \\
& V_{1}^{\prime}+\frac{1}{2} \alpha^{\prime} V_{1}+\frac{i}{2} e^{\alpha-\beta} V_{1} \cot \theta-i e^{\alpha}(m-D) V_{4}+E e^{\alpha} V_{2}=0,
\end{aligned}
$$

Let us remark that in order to solve the set of equations (24)-(27), we must determine $\mathrm{D}(\mathrm{S}, \mathrm{P})$ and $\mathrm{E}(\mathrm{S}, \mathrm{P})$ and then $\mathrm{S}$ and $\mathrm{P}$ as functions of $e^{\alpha(\xi)}$. In the following section, we shall resolve the fundamental fields equations. 122

\section{Results}

This section deals with the general solutions of the basic equations established in the previous section.

From (24)-(27), it is possible to write the equations for the functions $S=\bar{\psi} \psi, P=i \bar{\psi} \gamma^{5} \psi$ and $R=\bar{\psi} \bar{\gamma}^{5} \bar{\gamma}^{1} \psi$ as follows

$$
\begin{gathered}
S^{\prime}+\alpha^{\prime} S+2 E e^{\alpha} R=0 \\
R^{\prime}+\alpha^{\prime} R+2(m-D) e^{\alpha} P+2 E e^{\alpha} S=0 \\
P^{\prime}+\alpha^{\prime} P+2(m-D) e^{\alpha} R=0
\end{gathered}
$$

The first integral for the system (28)-(30) is

$$
P^{2}-R^{2}+S^{2}=A e^{-2 \alpha(\xi)}=-\frac{A}{g_{11}}, \quad A=\text { const } .
$$

The expression (31) comes to confirm that the spinor field of elementary particles and the own gravitational are linked by nature. Also, the same relation proves that the consideration of the proper gravitational field is very important in purpose to obtain solutions having the interest physics properties.

Let us now study the system of the invariant functions (28)- (30) considering massless elementary particles $(\mathrm{m}=0)$ and setting

$$
P_{0}(\xi)=e^{\alpha} P(\xi) ; S_{0}(\xi)=e^{\alpha} S(\xi) ; R_{0}(\xi)=e^{\alpha} R(\xi) .
$$

Inserting (32) into (28)-(30) we get the following system in $P_{0} ; S_{0}$ and $R_{0}$ :

$$
\begin{gathered}
S_{0}^{\prime}+2 E e^{\alpha} R_{0}=0 \\
R_{0}^{\prime}-2 D e^{\alpha} P_{0}+2 E e^{\alpha} S_{0}=0 \\
P_{0}^{\prime}-2 D e^{\alpha} R_{0}=0
\end{gathered}
$$

The previous system leads to the differential equation:

$$
P_{0} P_{0}^{\prime}-R_{0} R_{0}^{\prime}+S_{0} S_{0}^{\prime}=0 .
$$

The first integral of the equation (36) is

$$
P_{0}^{2}-R_{0}^{2}+S_{0}^{2}=A_{0},
$$

with $A_{0}$ being constant.

Then multiplying (33) by $\mathrm{D}(\mathrm{S}, \mathrm{P})$ and $(35)$ by $\mathrm{E}(\mathrm{S}, \mathrm{P})$ and combining the results, we obtain

$$
D S_{0}^{\prime}+E P_{0}^{\prime}=0
$$

Taking into account the expressions for $\mathrm{D}(\mathrm{S}, \mathrm{P})$ and $\mathrm{E}(\mathrm{S}, \mathrm{P})$ (23) we find

$$
2 S \frac{d G}{d I_{\nu}} S_{0}^{\prime}+2 P \frac{d G}{d I_{\nu}} P_{0}^{\prime}=0
$$

The equation (39) implies that

$$
S S_{0}^{\prime}+P P_{0}^{\prime}=0 \text {. }
$$

When we multiply (40) by $e^{\alpha(\xi)}$ and taking into account (16), we get the equation

$$
S_{0} S_{0}^{\prime}+P_{0} P_{0}^{\prime}=0
$$

which has the first integral given by the following relation

$$
P_{0}^{2}+S_{0}^{2}=A_{1}^{2}
$$

where $A_{1}$ being integration constant.

Since $P_{0}=e^{\alpha} P$ and $S_{0}=e^{\alpha} S$, from (32), we deduce

$$
I_{v}(\xi)=P^{2}+S^{2}=A_{1}^{2} e^{-2 \alpha(\xi)} .
$$

By substituting (42) into (31), we obtain

$$
R_{0}=\sqrt{A_{1}^{2}-A}, \quad \text { where } A_{1}^{2}-A>0 .
$$

It follows that

$$
R(\xi)=A_{2} e^{-\alpha(\xi)}, \text { with } A_{2}^{2}=A_{1}^{2}-A .
$$

Taking into account (23) and $P_{0}=\sqrt{A_{1}^{2}-S_{0}^{2}}$ due to (42), the expression (33) becomes

$$
\frac{d S_{0}}{d \xi}+4 \sqrt{A_{1}^{2}-S_{0}^{2}} \cdot A_{2} \frac{d G}{d I_{v}}=0
$$


The equation (46) has the first integral

$$
S_{0}(\xi)=-A_{1} \sin \Omega(\xi), S(\xi)=-A_{1} e^{-\alpha(\xi)} \sin \Omega(\xi)
$$

where

$$
\Omega(\xi)=4 A_{2} \int \frac{d G}{d I_{v}}+A_{3}, \quad A_{3}=\text { const }
$$

Introducing (47) into (42), we get

$$
P_{0}(\xi)=A_{1} \cos \Omega(\xi), P(\xi)=A_{1} e^{-\alpha(\xi)} \cos \Omega(\xi) .
$$

Using the spinor field equation in the form (21) and the conjugate one in the form (22), we obtain the following expression for $T_{1}^{1}$ from (16)

$$
T_{1}^{1}=m S-G\left(I_{v}\right)
$$

In the following paragraph, we shall solve the Einstein equations by determining the expressions of the metric functions $\alpha(\xi), \beta(\xi)$ and $\gamma(\xi)$. In view of $T_{0}^{0}=T_{2}^{2}$ for 13, the difference of Einstein equations (7) and 5 implies

$$
\beta^{\prime \prime}-\gamma^{\prime \prime}=e^{2 \beta+2 \gamma} \text {. }
$$

The equation (51) may be transformed to Liouville equation type [9]. Then, the equation (51) has the solutions

$$
\begin{gathered}
\beta(\xi)=\frac{H}{4}\left(1+\frac{2}{C}\right) \ln \frac{H}{C T^{2}\left(h, \xi+\xi_{1}\right)}=\left(1+\frac{2}{C}\right) \gamma(\xi) . \\
\gamma(\xi)=\frac{H}{4} \ln \frac{H}{C T^{2}\left(h, \xi+\xi_{1}\right)} .
\end{gathered}
$$

$\mathrm{H}$ and $\mathrm{C}$ being integration constants.

The function $\mathrm{T}$ has the form

$$
T\left(h, \xi+\xi_{1}\right)=\left\{\begin{array}{l}
\frac{1}{h} \sinh \left[h\left(\xi+\xi_{1}\right)\right], h>0 \\
\left(\xi+\xi_{1}\right), h=0 \\
\frac{1}{h} \sin \left[h\left(\xi+\xi_{1}\right)\right], h<0
\end{array}\right.
$$

$\mathrm{h}$ being an integration constant and $\xi_{1}$ another nonzero integration constant.

Taking into account (53), (54) and (3) we obtain the following expressions between the metric functions $\alpha(\xi), \beta(\xi)$ and $\gamma(\xi)$

$$
\begin{gathered}
\alpha(\xi)=\frac{H}{2}\left(\frac{3}{2}+\frac{2}{C}\right) \ln \frac{H}{C T^{2}\left(h, \xi+\xi_{1}\right)}, \\
\beta(\xi)=\frac{2+C}{4+3 C} \alpha(\xi) ; \quad \gamma(\xi)=\frac{C}{4+3 C} \alpha(\xi) .
\end{gathered}
$$

The equation (6) is the first integral of the equations (5) and (7). It is also a first order differential equation. Substituting (52) and (58) into (6), we get the following expression

$$
\left(\alpha^{\prime}\right)^{2}=\frac{(4+3 C)^{2}}{\left(3 C^{2}+8 C+4\right)} e^{2 \alpha}\left[e^{\frac{4+2 C}{4+3 C}(-\alpha)}-\chi\left(m S-G\left(I_{v}\right)\right)\right]
$$

For a concrete analytique form of the function $G\left(I_{v}\right)$, we can define the metric function $\alpha(\xi)$ from (57). Considering massless elementary particles, i.e. $m=0$, without losing the generality [11], the general analytical solutions of the equation (57) is

$$
\int \frac{d \alpha}{\frac{(4+3 C)}{\sqrt{3 C^{2}+8 C+4}} e^{\alpha} \sqrt{e^{\frac{4+2 C}{4+3 C}(-\alpha)}+\chi G\left(I_{v}\right)}}= \pm\left(\xi+\xi_{0}\right), \quad \xi_{0}=\text { const. }
$$

Let us now pass from $\alpha(\xi)$ to $I_{v}(\xi)$. Taking into account (43), we have

$$
e^{\alpha(\xi)}=\frac{A_{1}}{\sqrt{I_{v}}}, \quad d \alpha=-\frac{1}{2} \frac{d I_{v}}{I_{v}} .
$$

Inserting (59) into (58), one gets the following solution

$$
\int \frac{d I_{v}}{\frac{(4+3 C)}{\sqrt{3 C^{2}+8 C+4}} \sqrt{I_{v}} \sqrt{\left(\frac{I_{v}}{A_{1}^{2}}\right)^{\frac{2+C}{4+3 C}}+\chi G\left(I_{v}\right)}}= \pm 2 A_{1}\left(\xi+\xi_{0}\right), \quad \xi_{0}=\text { const. }
$$

Let us remark that, knowing the analytical form of $G\left(I_{v}\right)$, we can determine the analytic explicite form of the invariant function $I_{v}(\xi)$. Furthermore, we can determine the metric functions $\alpha(\xi), \beta(\xi)$ and $\gamma(\xi)$ from the equation(43) and (56) respectively as well as the functions $\mathrm{S}, \mathrm{P}, \mathrm{D}(\mathrm{S}, \mathrm{P})$ and $\mathrm{E}(\mathrm{S}, \mathrm{P})$. 
From (47) and (49), we define the functions $\mathrm{D}(\mathrm{S}, \mathrm{P})$ and $\mathrm{E}(\mathrm{S}, \mathrm{P})$ by the following relations

$$
\begin{aligned}
& D(S, P)=2 S \frac{d G\left(I_{v}\right)}{d I_{v}}=-2 C_{1} \sin \Omega(\xi) \cdot e^{-\alpha(\xi)} \frac{d G\left(I_{v}\right)}{d I_{v}} \\
& E(S, P)=2 P \frac{d G\left(I_{v}\right)}{d I_{v}}=2 C_{1} \cos \Omega(\xi) \cdot e^{-\alpha(\xi)} \frac{d G\left(I_{v}\right)}{d I_{v}}
\end{aligned}
$$

Introducing (61) and (62) into (24)-(27) and considering $m=0$ and $W_{\delta}(\Omega(\xi))=V_{\delta}(\xi) e^{\frac{\alpha}{2}}, \delta=1,2,3,4$ where $\Omega(\xi)$ is defined by (48), we get

$$
\begin{aligned}
& W_{4}^{\prime}(\Omega)-\Phi\left(I_{v}\right) W_{4}+i a \sin \Omega(\xi) W_{1}-a \cos \Omega(\xi) W_{3}=0 \\
& W_{3}^{\prime}(\Omega)+\Phi\left(I_{v}\right) W_{3}+i a \sin \Omega(\xi) W_{2}-a \cos \Omega(\xi) W_{4}=0 \\
& W_{2}^{\prime}(\Omega)-\Phi\left(I_{v}\right) W_{2}-i a \sin \Omega(\xi) W_{3}+a \cos \Omega(\xi) W_{1}=0 \\
& W_{1}^{\prime}(\Omega)+\Phi\left(I_{v}\right) W_{1}-i a \sin \Omega(\xi) W_{4}+a \cos \Omega(\xi) W_{2}=0
\end{aligned}
$$

With $\Phi\left(I_{v}\right)=\frac{i}{8 C_{2} \frac{d G}{d I_{v}}} \cot \theta, a=\frac{C_{1}}{2 C_{2}}$ and $W_{\delta}^{\prime}(\Omega)=\frac{d W_{\delta}}{d \Omega} . \quad$ yields the solution

Here for simplicity, let us pass to the new functions $U_{\delta}(\Omega)$ in the system (63)-(66):

$$
\begin{aligned}
& U_{1}=W_{1}+W_{2}+W_{3}+W_{4} \\
& U_{2}=W_{1}+W_{2}-W_{3}-W_{4} \\
& U_{3}=W_{1}-W_{2}+W_{3}-W_{4} \\
& U_{4}=W_{1}-W_{2}-W_{3}+W_{4}
\end{aligned}
$$

Inserting (67)-(70) into (63)-(66) and summing the results, we obtain the following set of equations:

$$
\begin{aligned}
& U_{4}^{\prime \prime}+i U_{4}^{\prime}-\left[a^{2}+\phi^{2}\left(I_{v}\right)\right] U_{4}+i \phi\left(I_{v}\right) U_{2}=0 \\
& U_{3}^{\prime \prime}+i U_{3}^{\prime}-\left[a^{2}+\phi^{2}\left(I_{v}\right)\right] U_{3}-i \phi\left(I_{v}\right) U_{1}=0 \\
& U_{2}^{\prime \prime}+i U_{2}^{\prime}-\left[a^{2}+\phi^{2}\left(I_{v}\right)\right] U_{2}+i \phi\left(I_{v}\right) U_{4}=0 \\
& U_{1}^{\prime \prime}+i U_{1}^{\prime}-\left[a^{2}+\phi^{2}\left(I_{v}\right)\right] U_{1}-i \phi\left(I_{v}\right) U_{3}=0
\end{aligned}
$$

Summing (71) and (73) and setting $X(\Omega)=U_{2}+U_{4}$, we have the differential equation

$$
X^{\prime \prime}+i X^{\prime}-\left[a^{2}+\phi^{2}\left(I_{v}\right)-i \phi\left(I_{v}\right)\right] X=0
$$

$$
U_{2}+U_{4}=K_{2} e^{r_{2} \Omega(\xi)}+K_{4} e^{r_{4} \Omega(\xi)}, K_{2}, \quad K_{4}=\text { const }
$$

with

$$
r_{2,4}=-\frac{i}{2} \pm \sqrt{a^{2}-\frac{1}{4}\left(1+\frac{\cot \theta}{4 C_{2} \frac{d G}{d I_{v}}}\right)^{2}}
$$

Substracting equations (71) and (73) and using $Y(\Omega)=$ $U_{2}-U_{4}$, we obtain the equation

$$
Y^{\prime \prime}+i Y^{\prime}-\left[a^{2}+\phi^{2}\left(I_{v}\right)+i \phi\left(I_{v}\right)\right] Y=0
$$

The solution of the equation (78) is given by the following expression

$$
U_{2}-U_{4}=K_{2}^{\prime} e^{r_{2}^{\prime} \Omega(\xi)}+K_{4}^{\prime} e^{r_{4}^{\prime} \Omega(\xi)}, K_{2}^{\prime}, K_{4}^{\prime}=\text { const } .
$$

where

$$
r_{2,4}^{\prime}=-\frac{i}{2} \pm \sqrt{a^{2}-\frac{1}{4}\left(1-\frac{\cot \theta}{4 C_{2} \frac{d G}{d I_{v}}}\right)^{2}}
$$

Taking the realtions (76) and (79) into account, we deduce the expressions of the functions $U_{2}$ and $U_{4}$ as follows

$$
\begin{aligned}
& U_{2}(\Omega)=\frac{1}{2}\left[K_{2} e^{r_{2} \Omega(\xi)}+K_{4} e^{r_{4} \Omega(\xi)}+K_{2}^{\prime} e^{r_{2}^{\prime} \Omega(\xi)}+K_{4}^{\prime} e^{r_{4}^{\prime} \Omega(\xi)}\right] \\
& U_{4}(\Omega)=\frac{1}{2}\left[K_{2} e^{r_{2} \Omega(\xi)}+K_{4} e^{r_{4} \Omega(\xi)}-K_{2}^{\prime} e^{r_{2}^{\prime} \Omega(\xi)}-K_{4}^{\prime} e^{r_{4}^{\prime} \Omega(\xi)}\right]
\end{aligned}
$$

We also obtain the differential equation of the function $\mathrm{Z}$ setting $Z(\Omega)=U_{1}+U_{3}$ and combining the equations (72) and (74)

$$
Z^{\prime \prime}-i Z^{\prime}-\left[a^{2}+\phi^{2}\left(I_{v}\right)+i \phi\left(I_{v}\right)\right] Z=0 .
$$


The equation (83) has solution

$$
U_{1}+U_{3}=K_{1} e^{r_{1} \Omega(\xi)}+K_{3} e^{r_{3} \Omega(\xi)}, \quad K_{1}, \quad K_{3}=\text { const }
$$

with

$$
r_{1,3}=\frac{i}{2} \pm \sqrt{a^{2}-\frac{1}{4}\left(1-\frac{\cot \theta}{4 C_{2} \frac{d G}{d I_{v}}}\right)^{2}}
$$

Choosing $M(\Omega)=U_{1}-U_{3}$, the difference of the equations (72) and (74) leads to

$$
M^{\prime \prime}-i M^{\prime}-\left[a^{2}+\phi^{2}\left(I_{v}\right)-i \phi\left(I_{v}\right)\right] M=0 .
$$

Its solution is

$$
U_{1}-U_{3}=K_{1}^{\prime} e^{r_{1}^{\prime} \Omega(\xi)}+K_{3}^{\prime} e^{r_{3}^{\prime} \Omega(\xi)}, \quad K_{1}^{\prime}, \quad K_{3}^{\prime}=\text { const }
$$

where

$$
r_{1,3}^{\prime}=\frac{i}{2} \pm \sqrt{a^{2}-\frac{1}{4}\left(1+\frac{\cot \theta}{4 C_{2} \frac{d G}{d I_{v}}}\right)^{2}}
$$

The expressions of the functions $U_{1}(\Omega)$ and $U_{3} \Omega$ ) are defined from (84) and (86) as follows

$$
\begin{aligned}
& U_{1}(\Omega)=\frac{1}{2}\left[K_{1} e^{r_{1} \Omega(\xi)}+K_{3} e^{r_{3} \Omega(\xi)}+K_{1}^{\prime} e^{r_{1}^{\prime} \Omega(\xi)}+K_{3}^{\prime} e^{r_{3}^{\prime} \Omega(\xi)}\right] \\
& U_{3}(\Omega)=\frac{1}{2}\left[K_{1} e^{r_{1} \Omega(\xi)}+K_{3} e^{r_{3} \Omega(\xi)}-K_{1}^{\prime} e^{r_{1}^{\prime} \Omega(\xi)}-K_{3}^{\prime} e^{r_{3}^{\prime} \Omega(\xi)}\right]
\end{aligned}
$$

Let us remark that as the functions $U_{\delta}(\Omega)$ are known, we can pass to the functions $W_{\delta}(\Omega)$ and then to the functions $V_{\delta}(\xi)$. Thus, we have found the general solutions to the set of equations (24)-(27) for $m=0$ containing eight integration constants $K_{1}$, $K_{2}, K_{3}, K_{4}, K_{1}^{\prime}, K_{2}^{\prime}, K_{3}^{\prime}$ and $K_{4}^{\prime}$ and an arbitrary function $G\left(I_{v}\right)$.

In the following section, we shall analyze the equation (60) in details given the concrete form of nonlinear terms in spinor lagrangian density.

\section{Discussion}

We choose the nonlinear terms in the lagrangian density in the form [11]:

$$
G\left(I_{v}\right)=\lambda I_{v}^{n}, \quad n>1
$$

where $\lambda$ and $\mathrm{n}$ are the parameters of nonlinearity and power of nonlinearity respectively. It is convenient to separately analyze the two cases $n=1$ and $n>1$.

For $n=1$ and $\lambda \neq 0$, we have Heisenberg-Ivanenko type nonlinear spinor field equation given by the following expression

$$
i e^{-\alpha} \bar{\gamma}^{1}\left(\partial_{\xi}+\frac{1}{2} \alpha^{\prime}\right) \psi+\frac{i}{2} \bar{\gamma}^{2} e^{-\beta} \psi \cot \theta-\left(m-2 \lambda I_{v} \bar{\psi} \psi\right) \psi-2 \lambda I_{v}(\bar{\psi} \psi) \psi=0
$$

Substituting $G\left(I_{v}\right)=\lambda I_{v}$ into (60) with $\frac{2+C}{4+3 C}=1$, we obtain

$$
I_{v}(\xi)=\exp \left[\frac{2 A_{1}^{2} \sqrt{1+\lambda \chi A_{1}^{2}(4+3 C)}}{\sqrt{\left(3 C^{2}+8 C+4\right)}}\left(\xi+\xi_{0}\right)\right]
$$

In this case, the energy density is given by the following expression

$$
T_{0}^{0}(\xi)=\lambda \exp \left[\frac{2 A_{1}^{2} \sqrt{1+\lambda \chi A_{1}^{2}(4+3 C)}}{\sqrt{\left(3 C^{2}+8 C+4\right)}}\left(\xi+\xi_{0}\right)\right]
$$


It then follows that the metric functions $\alpha(\xi), \beta(\xi)$ and $\gamma(\xi)$ are defined under the forms

$$
\begin{gathered}
\alpha(\xi)=\ln \left[\frac{A_{1}}{\sqrt{\exp \left[\frac{2 A_{1}^{2} \sqrt{1+\lambda \chi A_{1}^{2}(4+3 C)}}{\sqrt{\left(3 C^{2}+8 C+4\right)}}\left(\xi+\xi_{0}\right)\right]}}\right] \\
\beta(\xi)=\ln \left[\frac{A_{1}}{\sqrt{\exp \left[\frac{2 A_{1}^{2} \sqrt{1+\lambda \chi A_{1}^{2}(4+3 C)}}{\sqrt{\left(3 C^{2}+8 C+4\right)}}\left(\xi+\xi_{0}\right)\right]}}\right] \\
\gamma(\xi)=\left(\frac{C}{4+3 C}\right) \ln \left[\frac{A_{1}}{\sqrt{\exp \left[\frac{2 A_{1}^{2} \sqrt{1+\lambda \chi A_{1}^{2}(4+3 C)}}{\sqrt{\left(3 C^{2}+8 C+4\right)}}\left(\xi+\xi_{0}\right)\right]}}\right]
\end{gathered}
$$

From (94), the distribution of the spinor field energy density per unit invariant volume is

$$
\begin{aligned}
f(\xi) & =T_{0}^{0}(\xi) \sqrt{-3_{g}} \\
& =\lambda \exp \left[\frac{2 A_{1}^{2} \sqrt{1+\lambda \chi A_{1}^{2}(4+3 C)}}{\sqrt{\left(3 C^{2}+8 C+4\right)}}\left(\xi+\xi_{0}\right)\right] \varphi(\xi) \sin \theta
\end{aligned}
$$

where

$$
\varphi(\xi)=\left[\frac{A_{1}}{\sqrt{\exp \left[\frac{2 A_{1}^{2} \sqrt{1+\lambda \chi A_{1}^{2}(4+3 C)}}{\sqrt{\left(3 C^{2}+8 C+4\right)}}\left(\xi+\xi_{0}\right)\right]}}\right]^{\frac{8+5 C}{4+3 C}}
$$

Note that the equation (92) possesses soliton-like solutions when $G\left(I_{v}\right)=\lambda I_{v}$. Indeed $I_{v}(\xi)$ is a continuous and bounded function when $\xi \in\left[0, \xi_{c}\right]$, the quantities $g_{00}=e^{2 \gamma(\xi)}, g_{11}=-e^{2 \alpha(\xi)}, g_{22}=-e^{2 \beta(\xi)}$ and $g_{33}=-e^{2 \beta(\xi)} \sin ^{2} \theta$ are regular, the spinor field energy density is localized, according to (94), and the total energy $E=\int_{0}^{\xi_{c}} T_{0}^{0}(\xi) \sqrt{-3_{g}} d \xi$ is finite.

Then, for $n>1$ and $L_{N}=G\left(I_{v}\right)=\lambda I_{v}^{n}$ we have

$$
I_{v}(\xi)=\left\{\frac{1}{\sqrt{\chi \lambda A_{1}^{2}} \sinh \left[\frac{(4+3 C)}{\sqrt{3 C^{2}+8 C+4}}(n-1)\left(\xi+\xi_{0}\right)\right]}\right\}^{\frac{2}{n-1}} ; n>1 .
$$

As for the energy density, it is defined by the following expression

$$
T_{0}^{0}(\xi)=\lambda(2 n-1)\left\{\frac{1}{\sqrt{\chi \lambda A_{1}^{2}} \sinh \left[\frac{(4+3 C)}{\sqrt{3 C^{2}+8 C+4}}(n-1)\left(\xi+\xi_{0}\right)\right]}\right\}^{\frac{2 n}{n-1}} ; n>1 .
$$

From (99) and (100), when $\xi \longrightarrow 0, \xi_{0}=0$, we note that $I_{v}(\xi) \longrightarrow \infty$ and $T_{0}^{0}(\xi) \longrightarrow \infty$. It is clear from (100) that the energy density is not localized. In this condition the total energy is unlimited. Therefore, the soliton-like configurations are absent.

Let us consider the expression for $I_{v}(\xi)$ which follows from (40) when the nonlinearity of parameter $\lambda=-\Lambda^{2}<0$ and $n>1$. In this case, we obtain:

$$
I_{v}(\xi)=\left\{\frac{1}{\chi \Lambda^{2} A_{1}^{2} \cosh ^{2}\left[\frac{(4+3 C)}{\sqrt{3 C^{2}+8 C+4}}(n-1)\left(\xi+\xi_{0}\right)\right]}\right\}^{\frac{1}{n-1}} ; n>1 .
$$


We remark that $I_{v}(\xi)$ is a continuous and bounded function when $\xi \in\left[0, \xi_{c}\right]$.

Then, the energy density is defined by the following expression

$$
T_{0}^{0}(\xi)=-\Lambda^{2}(2 n-1)\left\{\frac{1}{\chi \Lambda^{2} A_{1}^{2} \cosh ^{2}\left[\frac{(4+3 C)}{\sqrt{3 C^{2}+8 C+4}}(n-1)\left(\xi+\xi_{0}\right)\right]}\right\}^{\frac{n}{n-1}} ; n>1 .
$$

Let us consider the energy density per unit volume invariant:

$$
\begin{aligned}
\Gamma(\xi) & =T_{0}^{0}(\xi) \sqrt{-3_{g}} \\
& =-\Lambda^{2}(2 n-1) \zeta(\xi)\left\{\frac{1}{\chi \Lambda^{2} A_{1}^{2} \cosh ^{2}\left[\frac{(4+3 C)}{\sqrt{3 C^{2}+8 C+4}}(n-1)\left(\xi+\xi_{0}\right)\right]}\right\}^{\frac{n}{n-1}} \sin \theta
\end{aligned}
$$

where

$$
\zeta(\xi)=\left[\frac{A_{1}}{\left\{\frac{1}{\chi \Lambda^{2} A_{1}^{2} \cosh ^{2}\left[\frac{(4+3 C)}{\sqrt{3 C^{2}+8 C+4}}(n-1)\left(\xi+\xi_{0}\right)\right]}\right\}^{\frac{1}{n-1}}}\right]^{\frac{8+5 C}{4+3 C}}
$$

Emphasize that the spinor field energy density per unit volume invariant $\Gamma(\xi)$ is localized and the total energy $E=\int_{0}^{\xi_{c}} \Gamma(\xi) d \xi$ has a finite quantity.

Let us find the explicit form of the functions $V_{\delta}(\xi), \delta=1,2,3,4$. To this end, we must determine an explicit form of $U_{\delta}(\xi)$, then $W_{\delta}(\xi)$ and subsequently $V_{\delta}(\xi)=W_{\delta}(\xi) e^{-\frac{1}{2} \alpha(\xi)}$. We obtain:

$$
\begin{aligned}
& V_{1}(\xi)=\frac{1}{4 \sqrt{A_{1}}}\left[K_{1} e^{r_{1} \Omega(\xi)}+K_{2} e^{r_{2} \Omega(\xi)}+K_{3} e^{r_{3} \Omega(\xi)}+K_{4} e^{r_{4} \Omega(\xi)}\right] v(\xi) \\
& V_{2}(\xi)=\frac{1}{4 \sqrt{A_{1}}}\left[K_{1}^{\prime} e^{r_{1}^{\prime} \Omega(\xi)}+K_{2}^{\prime} e^{r_{2}^{\prime} \Omega(\xi)}+K_{3}^{\prime} e^{r_{3}^{\prime} \Omega(\xi)}+K_{4}^{\prime} e^{r_{4}^{\prime} \Omega(\xi)}\right] v(\xi) \\
& V_{3}(\xi)=\frac{1}{4 \sqrt{A_{1}}}\left[K_{1} e^{r_{1} \Omega(\xi)}+K_{3} e^{r_{3} \Omega(\xi)}-K_{2} e^{r_{2} \Omega(\xi)}-K_{4} e^{r_{4} \Omega(\xi)}\right] v(\xi) \\
& V_{3}(\xi)=\frac{1}{4 \sqrt{A_{1}}}\left[K_{1}^{\prime} e^{r_{1}^{\prime} \Omega(\xi)}+K_{3}^{\prime} e^{r_{3}^{\prime} \Omega(\xi)}-K_{2}^{\prime} e^{r_{2}^{\prime} \Omega(\xi)}-K_{4}^{\prime} e^{r_{4}^{\prime} \Omega(\xi)}\right] v(\xi)
\end{aligned}
$$

where

and

$$
v(\xi)=\left\{\frac{1}{\sqrt{\chi \Lambda^{2} A_{1}^{2}} \cosh \left[\frac{(4+3 C)}{\sqrt{3 C^{2}+8 C+4}}(n-1)\left(\xi+\xi_{0}\right)\right]}\right\}^{\frac{1}{2 n-2}}
$$

$$
\Omega(\xi)=-\frac{4 n A_{2} \sqrt{3 C^{2}+8 C+4}}{\chi A_{1}^{2}(4+3 C)(n-1)} \tanh \left[\frac{(4+3 C)}{\sqrt{3 C^{2}+8 C+4}}(n-1)\left(\xi+\xi_{0}\right)\right]+A_{3} .
$$

The expression of the function $\Omega(\xi)$ is obtained by substituting $G\left(I_{v}\right)=-\Lambda^{2} I_{v}^{n}$ in (48).

The following paragraph addresses the total charge and the total spin.

To this end, according to the expressions (104)-(107), let us write the components of the spinor current vector $j^{\mu}=\bar{\psi} \gamma^{\mu} \psi$ :

$$
j^{0}=\bar{F}(\Omega) \cdot e^{-\alpha-\gamma}
$$

Since the configuration is static, the another components $j^{1}, j^{2}$ and $j^{3}$ are null. The component $j^{0}$ determines the charge density of the spinor field given by the expression:

$$
\varrho(\xi)=\left(j_{0} j^{0}\right)^{\frac{1}{2}}=\bar{F}(\Omega) \cdot e^{-\alpha}
$$


From (59), (91) and (110), we have

$$
\varrho(\xi)=\bar{F}(\Omega) A_{1}^{-1}\left\{\frac{1}{\chi \Lambda^{2} A_{1}^{2} \cosh ^{2}\left[\frac{(4+3 C)}{\sqrt{3 C^{2}+8 C+4}}(n-1)\left(\xi+\xi_{0}\right)\right]}\right\}^{\frac{1}{2(n-1)}}
$$

The charge density per unit invariant volume of the spinor field is defined by:

$$
q(\xi)=\varrho(\xi) \sqrt{-3_{g}}=\bar{F}(\Omega) A_{1}^{-1}\left\{\frac{1}{\chi \Lambda^{2} A_{1}^{2} \cosh ^{2}\left[\frac{(4+3 C)}{\sqrt{3 C^{2}+8 C+4}}(n-1)\left(\xi+\xi_{0}\right)\right]}\right\}^{\frac{1}{2(n-1)}} \zeta(\xi) \sin \theta
$$

The total charge of the spinor field is:

$$
Q(\xi)=\int_{0}^{\xi_{c}} q(\xi) d \xi
$$

$\xi_{C}$ being the center of the field configuration.

It follows that from (113) and (113) the charge density and the charge density per unit invariant volume are localized because the integrands $\varrho(\xi)$ and $q(\xi)$ are continuous and limited functions when $\xi \in\left[0, \xi_{c}\right]$. Moreover, the total charge is a finite quantity when the nonlinear term of the spinor field is choosen under the form $L_{N}=-\Lambda^{2} I_{v}^{n}$.

Then, the general expression of the spin tensor of the spinor field reads [12, 13]

$$
S^{\mu \nu, \varepsilon}=\frac{1}{4} \bar{\psi}\left\{\gamma^{\varepsilon} \sigma^{\mu \nu}+\sigma^{\mu \nu} \gamma^{\varepsilon}\right\} \psi
$$

where $\sigma^{\mu \nu}=\left(\frac{i}{2}\right)\left[\gamma^{\mu} \gamma^{\nu}-\gamma^{\nu} \gamma^{\mu}\right]$. The spatial density of the spin vector $S^{i k, 0}, i, \mathrm{k}=1,2,3$, is given by the following expression

$$
S^{i k, 0}=\frac{1}{4} \bar{\psi}\left\{\gamma^{0} \sigma^{i k}+\sigma^{i k} \gamma^{0}\right\} \psi=\frac{1}{2} \bar{\psi} \gamma^{0} \sigma^{i k} \psi
$$

From (113), we find the components of the spin tensor of the spinor field as follows

$$
S^{12,0}=0, S^{13,0}=0, S^{23,0}=\frac{1}{2} \bar{V}_{\delta} \gamma^{0} \sigma^{23} V_{\delta} \cdot e^{-\alpha}
$$

We remark that the only nontrivial component of the spin tensor is $S^{23,0}$. It defines the chronometric invariant spin tensor $S_{c h I}^{23,0}$ and the projection of spin vector $S_{1}$ on $\xi$ axis having the forms

$$
\begin{aligned}
S_{c h I}^{23,0} & =\left(S_{23,0} \cdot S^{23,0}\right)^{\frac{1}{2}}=\bar{F}(\Omega) e^{-\alpha} \\
S_{1} & =\int_{0}^{\xi_{c}} \bar{F}(\Omega) e^{-\alpha} \sqrt{3_{-} g} d \xi
\end{aligned}
$$

Let us remark that the integrands in (113) and (118) coincide. Thus, the total spin is also limited quantity as the total charge. As a result, the geometry of the metric, the nonlinearity of the spinor field and the own gravitaional field play an important role in order to obtain a soliton-like solutions with limited total charge and total spin. Theses results are consistent with experimentaly results obtained in the accelerator particles.

\section{Concluding Remarks}

In this paper, we have obtained analytics spherical symmetric solutions to the spinor and gravitational fields equations which are regular with a localized energy density and finite total energy only if the nonlinear terms in the spinor lagrangian are under the form $L_{N}=-\Lambda^{2} I_{v}^{n}$. It is necessary to introduce the nonlinear terms, describing the field selfinteractions in the lagrangian, to take into account the proper gravitational field of the elementary particles because without them, the total energy of the spinor field diverges. Here, the geometry of the metric doen't not ignore. Contrarely in the plane symmetric metric of space-time, in our research work, the total charge and the total spin are finite quantities. The following paper will deal with the nonlinear spinor field equations of the invariant $I_{T}=S^{2}-P^{2}$ in the gravitational theory.

\section{Conflicts of Interest}

The authors declare no conflicts of interest regarding the publication of this paper.

\section{References}

[1] Cadavid, A. C. and Finkelstein, R. J. (2001) J.Math.Physics, 42, 4419.

[2] Poplawski, N. J. (2009) Modern Physics Letters, 24, 431442.

[3] Ponomarev, V. N. and Obukhov, Yu. N., (1981) Gen.Relativ.Gavity, 13, 1037.

[4] Rybakov, Yu. P., Shikin, G. N. and Saha, B., (1997) Int.Theor.Phys., 36, 1475.

[5] Adomou, A. and Shikin, G. N. (1998) Izvestia VUZov, Fizika, 41, 69. 
[6] Adanhoumè, A., Adomou, A., Codo, F.P. and Hounkonnou, M.N. (2012) Journal of Modern Physics, 3, 935 . https://doi.org/10.4236/jmp.2012.39122

[7] saha, B. and Shikin, G. N. (2003) Czechoslovak Journal of Physics, 54, 597-620. https://doi.org/10.1023/B:CJOP.0000029690.61308.a5

[8] Adomou, A., Edou, J. and Massou, S. (2019) Journal of Modern Physics, 10, 1222-1234. https://doi.org/10.4236/jmp.2019.1010081

[9] Adomou, A., Edou, J. and Massou, S. (2019) Journal of Applied Mathematics and Physics, 7, 2018-2835. https://doi.org/10.4236/jamp.2019.711194

[10] Shikin, G. N. (1995) Theory of Solitons in General Relativity. URSS, Moscow.

[11] Heisenberg, W. , (1966) Introduction to Unified Field
Theory of Elementary Particles. Interscience Publishers, London.

[12] Adomou, A., Alvarado, R. and Shikin, G. N. (1995) Izvestiya Vuzov. Fizika, 863-868.

[13] Zhelnorovich, V. A. (1982) spinor Theory and Its applications in Physics and Mechanics. Nauka, Moscow.

[14] Bogoliliubov, N. N. and Shirkov, D. V. (1976) Introduction to the theory of Quantized Fields. Nauka, Moscow.

[15] Rainer Burghardt (2019) Journal of Modern Physics, 10, 1439-1453 . https://doi.org/10.4236/jmp.2019.1012096

[16] Slobodan Spremo (2019) Journal of Modern Physics, 10, 1532-1547 . https://doi.org/10.4236/jmp.2019.1013102

[17] Petkov, V. (2010) Minkowski spacetime: A Hundred Years Later, New York . https://doi.org/10.1007/978-90-481$3475-5$ 